\title{
Palladium-Catalyzed Arylation and
}

\section{Heteroarylation of Azolopyrimidines}

Javier Mendiola ${ }^{\dagger}$ Isabel Castellote ${ }^{\dagger}$, Julio Alvarez-Builla, ${ }^{\dagger}{ }^{J}$ avier Fernández-Gadea, $\mathcal{S}$

\author{
Antonio Gómez, $\mathcal{S}$ and Juan J. Vaquero ${ }^{*}$
}

Departamento de Química Orgánica, Universidad de Alcalá, 28871-Alcalá de Henares, Madrid, Spain. Lab. Janssen-Cilag, C/Río Jarama s/n, Polígono Industrial, 45007-Toledo, Spain 


\section{Supporting Information}

\section{CONTENTS}

-12 pages

-Experimental details and characterization data for compounds:

11, 13a-i, 14a, 14d, 14f-h, 15a,b, and 16a,b, 16d 
General Methods. Column chromatography was performed using silica gel $\left(60 \mathrm{~F}_{254}, 70\right.$ $200 \mathrm{~mm})$ as the stationary phase. All melting points are uncorrected. NMR spectra were obtained at 300 or $500 \mathrm{MHz}\left({ }^{1} \mathrm{H}\right)$ and $75 \mathrm{MHz}\left({ }^{13} \mathrm{C}\right)$. Chemical shifts are reported in ppm relative to tetramethylsilane. 5-Bromo-3-methoxycarbonylpyrimido[1,6-a]indole (5) and 5bromo-7-methoxycarbonyl-pyrido[3',2':4,5]pyrrolo[1,2-c]pyrimidine (6) were prepared according to the methods described in reference 9. 2-Methylthio-4-trimethylstannylpyrimidine employed in the synthesis of 16c was prepared following the methods described in reference 20 .

5-Bis(3-methoxycarbonylpyrimido[1,6-a]indole) (11). To a mixture of $100 \mathrm{mg}(0.328$ mmol) of 5 in dry DMF (4 mL) was added a solution of $62 \mathrm{mg}(0.164 \mathrm{mmol})$ of $\mathrm{Me}_{6} \mathrm{Sn}_{2}$ in dry DMF $(1 \mathrm{~mL})$ and $\mathrm{Pd}\left(\mathrm{PPh}_{3}\right)_{4}(5 \%)$. The mixture was heated to $80{ }^{\circ} \mathrm{C}$ under argon for $20 \mathrm{~h}$. After cooling, the reaction mixture was filtered through Celite and the Celite was washed with DMF and a mixture of $\mathrm{CH}_{2} \mathrm{Cl}_{2}: \mathrm{MeOH}(8: 2)$ saturated with $\mathrm{NH}_{3}$. The latter washes were evaporated under reduced pressure yielding $7.4 \mathrm{mg}$ of $11(5 \%)$ as an orange solid. $\mathrm{Mp}>300$ ${ }^{\circ} \mathrm{C}$; IR (KBr) $v_{\max } 3415,2924,1718,1459,1371,1257,1239 \mathrm{~cm}^{-1} ;{ }^{1} \mathrm{H}$ NMR $(500 \mathrm{MHz}$, $\left.\mathrm{CDCl}_{3}\right) \delta 3.91(\mathrm{~s}, 6 \mathrm{H}) ; 7.62-7.50(\mathrm{~m}, 4 \mathrm{H}) ; 7.67(\mathrm{~d}, 2 \mathrm{H}, J=7.7 \mathrm{~Hz}) ; 8.01(\mathrm{~s}, 2 \mathrm{H}) ; 8.18(\mathrm{~d}, 2 \mathrm{H}$, $J=7.5 \mathrm{~Hz}$ ); 9.31 (s, 2H); MS (EI) $m / z$ (relative intensity) $450\left(\mathrm{M}^{+}, 100\right) ; 199$ (54); 165 (59). Anal. Calcd for $\mathrm{C}_{26} \mathrm{H}_{18} \mathrm{~N}_{4} \mathrm{O}_{4}$ (450.46): C, 69.33; H, 4.03; N, 12.44. Found: C, 69.65; H, 3.76; N, 12.01.<smiles>COC(=O)c1cc2c(-c3c4ccccc4n4cnc(C(=O)OC)cc34)c3ccccc3n2cn1</smiles>

Synthesis of derivatives 13. General procedure. To a mixture of $5(1.0 \mathrm{mmol})$, the aryl or heteroaryl boronic acid $(1.0 \mathrm{mmol})$ and $\mathrm{K}_{2} \mathrm{CO}_{3}(192 \mathrm{mg}, 1.4 \mathrm{mmol})$ in toluene/MeOH $(20: 1)$ 
$(25 \mathrm{~mL})$ was added $\mathrm{Pd}\left(\mathrm{PPh}_{3}\right)_{4}(57 \mathrm{mg}, 0.049 \mathrm{mmol})$ under argon and the mixture was heated under reflux for $10 \mathrm{~h}$. The reaction mixture was cooled and the resulting precipitate was filtered off and washed with toluene and EtOAc. The solid was dissolved in $\mathrm{CH}_{2} \mathrm{Cl}_{2}$ and the organic solution washed with water and dried over $\mathrm{Na}_{2} \mathrm{SO}_{4}$. The organic phase was filtered through Celite, the Celite was washed with $\mathrm{CH}_{2} \mathrm{Cl}_{2}$ and the combined organic phases evaporated under reduced pressure to afford pure compounds $\mathbf{1 3} \mathbf{a}-\mathbf{j}$.

3-Methoxycarbonyl-5-phenylpyrimido[1,6-a]indole (13a). 85\%; yellow solid; mp 278$279{ }^{\circ} \mathrm{C}$; IR (KBr) $v_{\max } 1718,1458,1430,1368,1296,1231,1085 \mathrm{~cm}^{-1} ;{ }^{1} \mathrm{H}$ NMR $(500 \mathrm{MHz}$, $\left.\mathrm{CDCl}_{3}\right) \delta 4.00(\mathrm{~s}, 3 \mathrm{H}) ; 7.46-7.39(\mathrm{~m}, 1 \mathrm{H}) ; 7.70-7.53(\mathrm{~m}, 6 \mathrm{H}) ; 8.12-8.03(\mathrm{~m}, 2 \mathrm{H}) ; 8.41(\mathrm{~d}, 1 \mathrm{H}$, $J=1.4 \mathrm{~Hz}), 9.24(\mathrm{~d}, 1 \mathrm{H}, J=1.4 \mathrm{~Hz}) ;{ }^{13} \mathrm{C} \mathrm{NMR}\left(75 \mathrm{MHz}_{\mathrm{CDCl}}\right) \quad \delta 165.2 ; 138.4 ; 134.3$; $132.6 ; 129.6 ; 129.1 ; 128.8 ; 127.2 ; 125.4 ; 123.7 ; 120.4 ; 117.2 ; 113.0 ; 110.8 ; 52.8 ;$ MS (EI) $\mathrm{m} / z$ (relative intensity) $302\left(\mathrm{M}^{+}, 100\right) ; 242(20)$. Anal. Calcd for $\mathrm{C}_{19} \mathrm{H}_{14} \mathrm{~N}_{2} \mathrm{O}_{2}$ : C, 75.44; H, 4.69; N, 9.26. Found: C, 75.48; H, 4.67; N, 9.27.

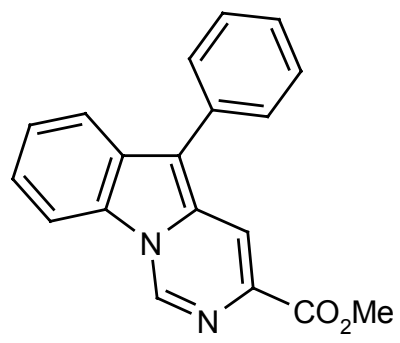

5-(4-Methylphenyl)-3-methoxycarbonylpyrimido[1,6-a]indole (13b). 68\%; orange solid; mp 283-285 ${ }^{\circ} \mathrm{C}$; IR (KBr) $v_{\max } 2953,1727,1428,1433,1370,1230,1090 \mathrm{~cm}^{-1} ;{ }^{1} \mathrm{H}$ $\operatorname{NMR}\left(500 \mathrm{MHz}, \mathrm{CDCl}_{3}\right) \delta 2.45(\mathrm{~s}, 3 \mathrm{H}) ; 3.97$ (s, 3H); $7.36(\mathrm{~d}, 2 \mathrm{H}, J=8.1 \mathrm{~Hz}) ; 7.56-7.50$ (m, 4H); 8.09-8.01 (m, 2H), 8.38 (d, 1H, $J=1.3 \mathrm{~Hz})$; 9.20 (d, 1H, $J=1.3 \mathrm{~Hz})$; Anal. Calcd for $\mathrm{C}_{20} \mathrm{H}_{16} \mathrm{~N}_{2} \mathrm{O}_{2}$ (316.36): C, 75.93; H, 5.10; N, 8.80. Found: C, 75.67; H, 4.89; N, 8.46.

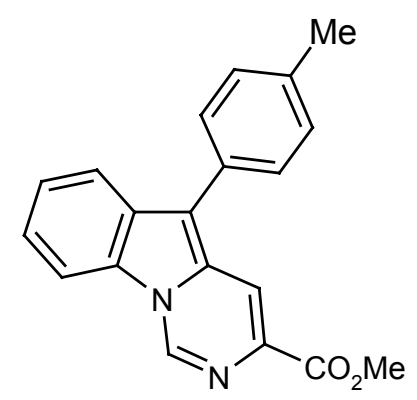


3-Methoxycarbonyl-5-(4-vinylphenyl)pyrimido[1,6-a]indole (13c). 56\%; orange solid; mp 275-276 ${ }^{\circ} \mathrm{C}$; IR (KBr) $v_{\max } 1720,1458,1433,1370,1295,1231,1091 \mathrm{~cm}^{-1} ;{ }^{1} \mathrm{H}$ NMR (500 $\left.\mathrm{MHz}, \mathrm{CDCl}_{3}\right) \delta 4.00(\mathrm{~s}, 3 \mathrm{H}) ; 5.33(\mathrm{~d}, 1 \mathrm{H}, J=10.9 \mathrm{~Hz}) ; 5.86(\mathrm{~d}, 1 \mathrm{H}, J=17.5 \mathrm{~Hz}) ; 6.82(\mathrm{dd}$, $1 \mathrm{H}, J=17.5,10.9 \mathrm{~Hz}) ; 7.68-7.53(\mathrm{~m}, 6 \mathrm{H}) ; 8.13-8.03(\mathrm{~m}, 2 \mathrm{H}) ; 8.41$ (d, $1 \mathrm{H}, J=1.4 \mathrm{~Hz}) ; 9.24$ (d, $1 \mathrm{H}, J=1.4 \mathrm{~Hz}$ ). Anal. Calcd for $\mathrm{C}_{21} \mathrm{H}_{16} \mathrm{~N}_{2} \mathrm{O}_{2}$ (328.37): C, 76.81; H, 4.91; N, 8.53. Found: C, $76.41 ; \mathrm{H}, 4.63 ; \mathrm{N}, 8.88$.

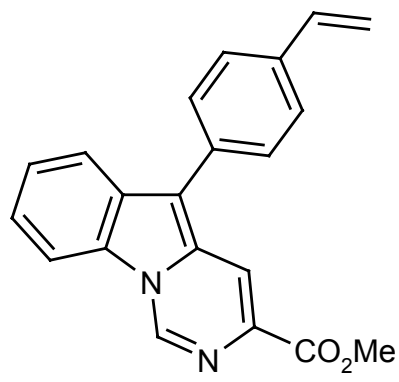

3-Methoxycarbonyl-5-(4-methoxyphenyl)pyrimido[1,6-a]indole (13d). $49 \%$; orangeyellowish solid; $\operatorname{mp} 285-286^{\circ} \mathrm{C}$; IR (KBr) $v_{\max } 1713,1457,1432,1369,1252,1231,1178$, $1088 \mathrm{~cm}^{-1} ;{ }^{1} \mathrm{H}$ NMR (500 MHz, $\left.\mathrm{CDCl}_{3}\right) \delta 3.91$ (s, 3H); 3.99 (s, 3H); 7.11 (d, 2H, J=8.7 Hz); 7.61-7.51 (m, 2H); 7.59 (d, 2H, J=8.7 Hz); 8.11-7.99 (m, 2H); 8.37 (d, 1H, $1.5 \mathrm{~Hz}) ; 9.21$ (d, $1 \mathrm{H}, J=1.5 \mathrm{~Hz}$ ). Anal. Calcd for $\mathrm{C}_{20} \mathrm{H}_{16} \mathrm{~N}_{2} \mathrm{O}_{3}$ (332.36): C, 72.28; H, 4.85; N, 8.43. Found: C, $72.67 ; \mathrm{H}, 5.02 ; \mathrm{N}, 8.64$.

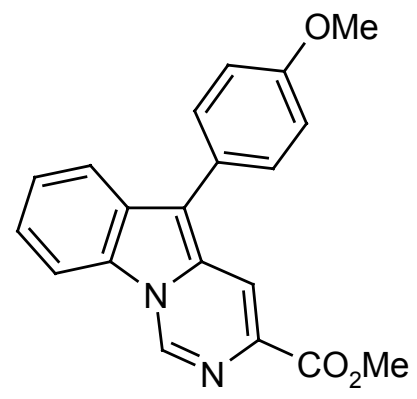

5-(4-Methylthiophenyl)-3-methoxycarbonylpyrimido[1,6-a]indole (13e). 71\%; orange solid; mp 302-303 ${ }^{\circ} \mathrm{C}$; IR (KBr) $v_{\max } 1717,1457,1432,1370,1295,1232,1092,1083 \mathrm{~cm}^{-1}$; 
${ }^{1} \mathrm{H}$ NMR $\left(500 \mathrm{MHz}, \mathrm{CDCl}_{3}\right) \delta 2.58$ (s, 3H); 4.00 (s, 3H); 7.46 (d, $\left.2 \mathrm{H}, J=8.4 \mathrm{~Hz}\right) ; 7.57-7.51$ (m, 2H); 7.59 (d, 2H, $J=8.4 \mathrm{~Hz}) ; 8.04-8.01(\mathrm{~m}, 1 \mathrm{H}) ; 8.11-8.08(\mathrm{~m}, 1 \mathrm{H}) ; 8.38(\mathrm{~d}, 1 \mathrm{H}, J=1.5$ $\mathrm{Hz}$ ); $9.23\left(\mathrm{~d}, 1 \mathrm{H}, J=1.5 \mathrm{~Hz}\right.$ ). Anal. Calcd for $\mathrm{C}_{20} \mathrm{H}_{16} \mathrm{~N}_{2} \mathrm{O}_{2}$ (316.36): C, 75.93; $\mathrm{H}, 5.10 ; \mathrm{N}$, 8.85. Found: C, $75.65 ; \mathrm{H}, 4.90 ; \mathrm{N}, 8.37$.

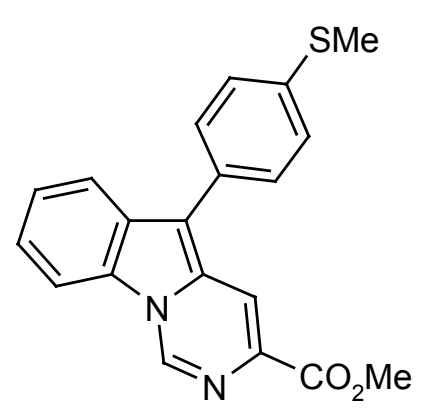

3-Methoxycarbonyl 5-(3,5-dichlorophenyl)pyrimido[1,6-a]indole (13f). 87\%; yellow solid; mp 302-303 ${ }^{\circ} \mathrm{C}{ }^{\circ} \mathrm{C}$; IR (KBr) $v_{\max } 1741,1586,1452,1433,1348,1224,1098 \mathrm{~cm}^{-1} ;{ }^{1} \mathrm{H}$ NMR (500 MHz, $\left.\mathrm{CDCl}_{3}\right) \delta 4.02(\mathrm{~s}, 3 \mathrm{H}) ; 7.53(\mathrm{~d}, 2 \mathrm{H}, J=1.8 \mathrm{~Hz}) ; 7.42(\mathrm{t}, 1 \mathrm{H}, J=1.8 \mathrm{~Hz})$, 7.60-7.56 (m, 2H); 8.01-7.96 (m, 1H), 8.14-8.09 (m, 1H), 8.34 (d, 1H, $J=1.5 \mathrm{~Hz}) ; 9.26(\mathrm{~d}$, $1 \mathrm{H}, J=1.5 \mathrm{~Hz}$ ). Anal. Calcd for $\mathrm{C}_{19} \mathrm{H}_{12} \mathrm{Cl}_{2} \mathrm{~N}_{2} \mathrm{O}_{2}$ (371.23): C, 61.48; H, 3.26; $\mathrm{N}, 7.55$. Found: C, $61.11 ; \mathrm{H}, 2.96 ; \mathrm{N}, 7.13$<smiles>COc1cc2c(-c3cc(Cl)cc(Cl)c3)c3ccccc3n2cn1</smiles>

3-Methoxycarbonyl-5-(4-formilphenyl)pyrimido[1,6-a]indole (13g).72\%; yellow solid; mp 294-295 ${ }^{\circ} \mathrm{C}$; IR (KBr) $v_{\max } 1710,1602,1526,1457,1432,1365,1300,1261,1235,1216$, 
$1164 \mathrm{~cm}^{-1} ;{ }^{1} \mathrm{H}$ NMR $\left(500 \mathrm{MHz}, \mathrm{CDCl}_{3}\right) \delta 4.02(\mathrm{~s}, 3 \mathrm{H}) ; 7.65-7.55(\mathrm{~m}, 2 \mathrm{H}) ; 7.86(\mathrm{~d}, 2 \mathrm{H}, J=$ $8.2 \mathrm{~Hz}$ ); 8.09 (d, 2H, $J=8.2 \mathrm{~Hz}) ; 8.16-8.05$ (m, 2H); 8.43 (d, 1H, $J=1.3 \mathrm{~Hz}) ; 9.29$ (d, 1H, $J$ $=1.3 \mathrm{~Hz}) ; 10.11(\mathrm{~s}, 1 \mathrm{H})$;. Anal. Calcd for $\mathrm{C}_{20} \mathrm{H}_{14} \mathrm{~N}_{2} \mathrm{O}_{3}(330.35)$ : C, 72.72; H, 4.27; N, 8.48. Found: C, 72.56; H, 4.45; N, 8.81.

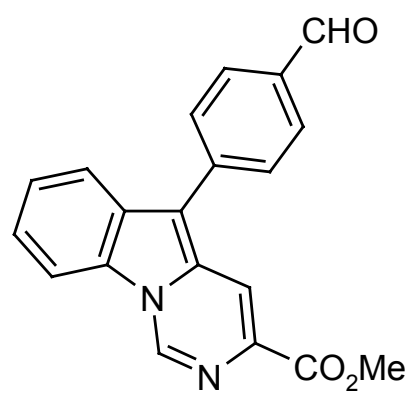

5-(Thiofen-3-yl)-3-methoxycarbonylpyrimido[1,6-a]indole (13h). 89\%; mp 294-295 ${ }^{\circ} \mathrm{C}$; IR (KBr) $v_{\max } 1716,1459,1426,1366,1294,1259,1234,1085 \mathrm{~cm}^{-1} ;{ }^{1} \mathrm{H}$ NMR $(500 \mathrm{MHz}$, $\left.\mathrm{CDCl}_{3}\right) \delta 4.01(\mathrm{~s}, 3 \mathrm{H}) ; 7.59-7.48(\mathrm{~m}, 5 \mathrm{H}) ; 8.11-8.03(\mathrm{~m}, 2 \mathrm{H}) ; 8.42(\mathrm{~d}, 1 \mathrm{H}, J=1.4 \mathrm{~Hz}) ; 9.21$ (d, $1 \mathrm{H}, J=1.4 \mathrm{~Hz}$ ). Anal. Calcd for $\mathrm{C}_{17} \mathrm{H}_{12} \mathrm{~N}_{2} \mathrm{O}_{2} \mathrm{~S}$ (308.36): C, 66.22; H, 3.92; N, 9.00. Found: C, 65.90; H, 3.74; N, 9.05.

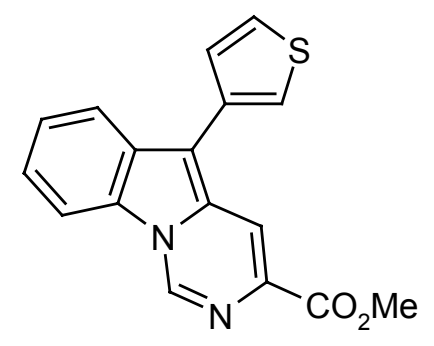

3-Methoxycarbonyl-5-(2-benzo[b]thiophenyl)pyrimido[1,6-a]indole (13i). 97\%; orange solid; mp $>300{ }^{\circ} \mathrm{C}$; IR (KBr) $v_{\max } 1711,1459,1431,1369,1230,1104 \mathrm{~cm}^{-1} ;{ }^{1} \mathrm{H}$ NMR (500 $\left.\mathrm{MHz}, \mathrm{CDCl}_{3}\right) \delta 4.00(\mathrm{~s}, 3 \mathrm{H}) ; 7.45-7.30(\mathrm{~m}, 2 \mathrm{H}) ; 7.65-7.50(\mathrm{~m}, 3 \mathrm{H}) ; 7.92-7.82(\mathrm{~m}, 2 \mathrm{H}) ; 8.10-$ $8.04(\mathrm{~m}, 1 \mathrm{H}) ; 8.28-8.23(\mathrm{~m}, 1 \mathrm{H}) ; 8.63(\mathrm{~d}, 1 \mathrm{H}, J=1.2 \mathrm{~Hz}) ; 9.21(\mathrm{~d}, 1 \mathrm{H}, J=1.2 \mathrm{~Hz})$. Anal. Calcd for $\mathrm{C}_{2} \mathrm{H}_{14} \mathrm{~N}_{2} \mathrm{O}_{2} \mathrm{~S}$ (358.42): C, 70.37; H, 3.94; N, 7.82. Found: C, 70.09; H, 3.69; N, 7.53 . 


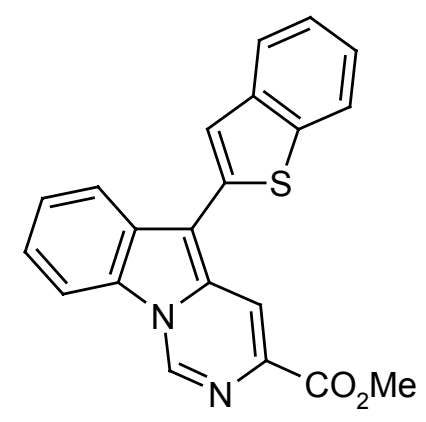

5-(2-Benzo[b]furanyl)-3-methoxycarbonylpyrimido[1,6-a]indole (13j). 67\%. mp 277$278{ }^{\circ} \mathrm{C}$; IR (KBr) $v_{\max } 1718,1453,1431,1367,1234 \mathrm{~cm}^{-1} ;{ }^{1} \mathrm{H}$ NMR $\left(500 \mathrm{MHz}, \mathrm{CDCl}_{3}\right) \delta$ 4.05 (s, 3H); 7.13 (s, 1H); 7.36-7.28 (m, 2H); 7.67-7.53 (m, 4H); 8.06 (d, 1H, J=8.2 Hz); $8.29(\mathrm{~d}, 1 \mathrm{H}, J=8.0 \mathrm{~Hz}) ; 8.87$ (d, $1 \mathrm{H}, J=1.5 \mathrm{~Hz}) ; 9.20(\mathrm{~d}, 1 \mathrm{H}, J=1.5 \mathrm{~Hz})$. Anal. Calcd for $\mathrm{C}_{21} \mathrm{H}_{14} \mathrm{~N}_{2} \mathrm{O}_{3}$ (342.36): C, 73.68; H, 4.12, N, 8.18. Found: C, 73.35; H, 3.86, N, 7.79.

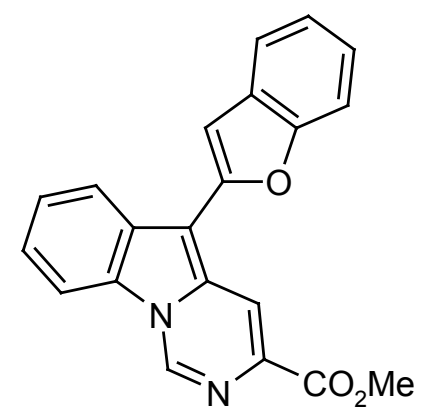

Synthesis of derivatives 14. General procedure. To a mixture of $6(1.0 \mathrm{mmol})$, the aryl or heteroaryl boronic acid $(1.0 \mathrm{mmol})$ and $\mathrm{K}_{2} \mathrm{CO}_{3}(192 \mathrm{mg}, 1.4 \mathrm{mmol})$ in toluene/MeOH $(20: 1)$ $(25 \mathrm{~mL})$ was added $\mathrm{Pd}\left(\mathrm{PPh}_{3}\right)_{4}(57 \mathrm{mg}, 0.049 \mathrm{mmol})$ under argon and the mixture was heated to reflux temperature for $10 \mathrm{~h}$. The reaction mixture was allowed to cool to room temperature, filtered through Celite, the Celite washed with $\mathrm{CH}_{2} \mathrm{Cl}_{2}$ and the organic phase evaporated under reduced pressure. The residue was purified by silica gel flash chromatography $\left[\mathrm{CH}_{2} \mathrm{Cl}_{2}\right.$ :acetone (95:5)] to afford pure compounds 14 . 
7-Methoxycarbonyl-5-phenylpyrido[3',2':4,5]pyrrolo[1,2-c]pyrimidine (14a). 66\%; yellow solid; Mp 234-235 ${ }^{\circ} \mathrm{C}$; IR (KBr) $v_{\max } 3414,2953,1711,1445,1289,1259,1092 \mathrm{~cm}^{-1}$; ${ }^{1} \mathrm{H}$ NMR $\left(500 \mathrm{MHz}, \mathrm{CDCl}_{3}\right) \delta 4.03(\mathrm{~s}, 3 \mathrm{H}) ; 7.70-7.42(\mathrm{~m}, 6 \mathrm{H}) ; 8.39$ (dd, $\left.1 \mathrm{H}, J=8.2,1.5 \mathrm{~Hz}\right)$; $8.46(\mathrm{~d}, 1 \mathrm{H}, J=1.5 \mathrm{~Hz}) ; 8.65(\mathrm{dd}, 1 \mathrm{H}, J=4.6,1.5 \mathrm{~Hz}) ; 9.65(\mathrm{~d}, 1 \mathrm{H}, J=1.5 \mathrm{~Hz}) ;{ }^{13} \mathrm{C} \mathrm{NMR}$ $\left(75 \mathrm{MHz} \mathrm{CDCl}_{3}\right) \quad \delta 53.0 ; 110.6 ; 116.9 ; 121.3 ; 127.4 ; 128.7 ; 128.8 ; 128.9 ; 129.3 ; 132.1 ;$ 138.3; 140.7; 144.6; 165.1; MS (EI) $m / z$ (relative intensity) $303\left(\mathrm{M}^{+}, 100\right)$; 245 (60). Anal. Calcd for $\mathrm{C}_{18} \mathrm{H}_{13} \mathrm{~N}_{3} \mathrm{O}_{2}$ (303.32): C, 71.27; H, 4.32; N,13.84. Found: C, 71.28; H, 4.32; N, 13.85

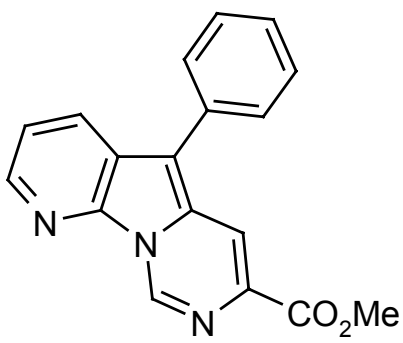

\section{5-(4-Methoxyphenyl)-7-methoxycarbonylpyrido $\left[3^{\prime}, 2^{\prime}: 4,5\right]$ pyrrolo $[1,2-c] p y r i m i d i n e$}

(14d). 52\%; orange solid. Mp 236-237 ${ }^{\circ} \mathrm{C}$; IR (KBr) $v_{\max } 3415,1711,1535,1436,1332$, 1294, $1259 \mathrm{~cm}^{-1} ;{ }^{1} \mathrm{H}$ NMR $\left(500 \mathrm{MHz}, \mathrm{CDCl}_{3}\right) \delta 3.91(\mathrm{~s}, 3 \mathrm{H}) ; 4.01(\mathrm{~s}, 3 \mathrm{H}), 7.11(\mathrm{dd}, 2 \mathrm{H}, J=$ $8.8 \mathrm{~Hz}) ; 7.53(\mathrm{dd}, 1 \mathrm{H}, J=8.2,4.6 \mathrm{~Hz}) ; 7.57$ (d, 2H, $J=8.8 \mathrm{~Hz}) ; 8.35(\mathrm{dd}, 1 \mathrm{H}, J=8.2,1.5$ $\mathrm{Hz}) ; 8.41(\mathrm{~d}, 1 \mathrm{H}, J=1.5 \mathrm{~Hz}) ; 8.63(\mathrm{dd}, 1 \mathrm{H}, J=4.6,1.5 \mathrm{~Hz}) ; 9.62(\mathrm{~d}, 1 \mathrm{H}, J=1.5 \mathrm{~Hz}) ;{ }^{13} \mathrm{C}$ NMR $\left(75 \mathrm{MHz} \mathrm{CDCl}_{3}\right) \delta 52.9 ; 55.4 ; 110.5 ; 114.9 ; 117.2 ; 121.2 ; 121.4 ; 124.5 ; 128.6 ; 128.8$; $130.1 ; 135.6 ; 138.4 ; 140.7 ; 144.6 ; 159.1 ; 165.3$. Anal. Calcd for $\mathrm{C}_{19} \mathrm{H}_{15} \mathrm{~N}_{3} \mathrm{O}_{3}$ (333.35): C, 68.46; H, 4.54; N, 12.61. Found: C, 68.67; H, 4.59; N, 12.50.

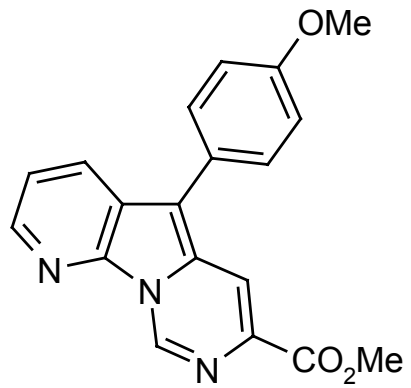




\section{5-(3,5-Dichlorophenyl)-7-methoxycarbonylpyrido[3',2':4,5]pyrrolo[1,2-c]pyrimidine}

(14f). 60\%; yellow solid. Mp 279-280 ${ }^{\circ} \mathrm{C}$; IR (KBr) $v_{\max } 3416,3049,1733,1439,1232 \mathrm{~cm}^{-1}$; ${ }^{1} \mathrm{H} \mathrm{NMR}\left(500 \mathrm{MHz}, \mathrm{CDCl}_{3}\right) \delta 4.03(\mathrm{~s}, 3 \mathrm{H}) ; 7.42(\mathrm{t}, 1 \mathrm{H}, J=1.8 \mathrm{~Hz}) ; 7.50$ (d, 2H, $\left.J=1.8 \mathrm{~Hz}\right)$; $7.57(\mathrm{dd}, 1 \mathrm{H}, J=8.2,4.6 \mathrm{~Hz}) ; 8.32(\mathrm{dd}, 1 \mathrm{H}, J=8.2,1.5 \mathrm{~Hz}) ; 8.38(\mathrm{~d}, 1 \mathrm{H}, J=1.5 \mathrm{~Hz}) ; 8.65$ $(\mathrm{dd}, 1 \mathrm{H} ; J=4.6,1.5 \mathrm{~Hz}) ; 9.67$ (d, $1 \mathrm{H}, J=1.5 \mathrm{~Hz}) ;{ }^{13} \mathrm{C} \mathrm{NMR}\left(75 \mathrm{MHz} \mathrm{CDCl}_{3}\right) \delta 53.1 ; 107.6$; $116.0 ; 121.0 ; 121.8 ; 127.0 ; 127.5 ; 128.3 ; 129.7 ; 135.3 ; 135.9 ; 137.1 ; 138.5 ; 140.8 ; 145.2$; 164.9. Anal. Calcd for $\mathrm{C}_{18} \mathrm{H}_{11} \mathrm{ClN}_{3} \mathrm{O}_{2}$ (372.21): C, 58.09; H, 2.98, N, 11.29. Found: C, 58.23; $\mathrm{H}, 2.65, \mathrm{~N}, 11.31$.

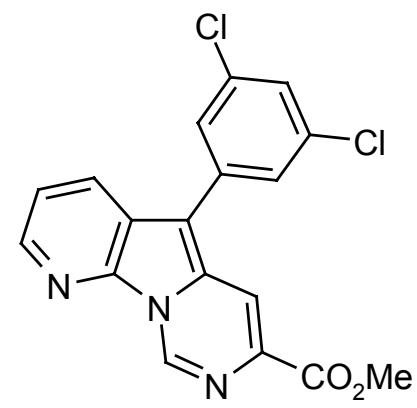

\section{7-Methoxycarbonyl-5-(4-formylphenyl)pyrido[3',2':4,5]pyrrolo[1,2-c]pyrimidine}

(14g). 65\%; orange solid; mp 246-247 ${ }^{\circ} \mathrm{C}$; IR (KBr) $v_{\max } 1710,1693,1442,1294,1161 \mathrm{~cm}^{-1}$; ${ }^{1} \mathrm{H}$ NMR $\left(500 \mathrm{MHz}, \mathrm{CDCl}_{3}\right) \delta 4.04(\mathrm{~s}, 3 \mathrm{H}) ; 7.59(\mathrm{dd}, 1 \mathrm{H}, J=8.2,4.6 \mathrm{~Hz}) ; 7.85(\mathrm{~d}, 2 \mathrm{H}, J=$ $8.2 \mathrm{~Hz}) ; 8.10(\mathrm{~d}, 2 \mathrm{H}, J=8.2 \mathrm{~Hz}) ; 8.41(\mathrm{dd}, 1 \mathrm{H}, J=8.2,1.5 \mathrm{~Hz}) ; 8.48(\mathrm{~d}, 1 \mathrm{H}, J=1.5 \mathrm{~Hz})$; $8.67(\mathrm{dd}, 1 \mathrm{H}, J=4.6,1.5 \mathrm{~Hz}) ; 9.70(\mathrm{~d}, 1 \mathrm{H}, J=1.5 \mathrm{~Hz}) ; 10.11(\mathrm{~s}, 1 \mathrm{H}) ;{ }^{13} \mathrm{C} \mathrm{NMR}(75 \mathrm{MHz}$ $\left.\mathrm{CDCl}_{3}\right) \delta 53.1 ; 109.1 ; 116.3 ; 120.9 ; 121.8 ; 128.5 ; 129.1 ; 129.8 ; 130.7 ; 134.9 ; 137.0 ; 138.5 ;$ 138.7; 140.9; 145.1; 164.8; 191.4. Anal. Calcd for $\mathrm{C}_{19} \mathrm{H}_{13} \mathrm{~N}_{3} \mathrm{O}_{3}(331.33)$ : C, 68.88; H, 3.95; N, 12.68. Found: C, 68.74; H, 3.91; N, 12.58 .

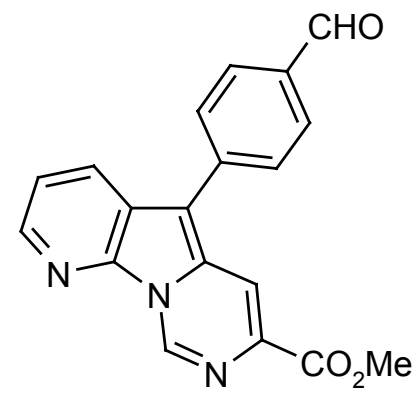


5-(Thiophen-3-yl)-7-methoxycarbonylpyrido[3',2':4,5]pyrrolo[1,2-c]pyrimidine (14h). 40\%; orange-yellowish solid; mp 258-259 ${ }^{\circ} \mathrm{C}$; IR (KBr) $v_{\max } 3415,1711,1436,1332,1258$ $\mathrm{cm}^{-1} ;{ }^{1} \mathrm{H}$ NMR $\left(500 \mathrm{MHz}, \mathrm{CDCl}_{3}\right) \delta 4.02(\mathrm{~s}, 3 \mathrm{H}) ; 7.48(\mathrm{dd}, 1 \mathrm{H}, J=8.2,4.6 \mathrm{~Hz}), 7.60-7.53$ $(\mathrm{m}, 3 \mathrm{H}), 8.63(\mathrm{dd}, 1 \mathrm{H}, J=8.2,1.5 \mathrm{~Hz}) ; 8.43(\mathrm{~d}, 1 \mathrm{H}, J=1.5 \mathrm{~Hz}) ; 8.63(\mathrm{dd}, 1 \mathrm{H}, J=4.6,1.5$ $\mathrm{Hz}) ; 9.63(\mathrm{~d}, 1 \mathrm{H}, J=1.5 \mathrm{~Hz})$; Anal. Calcd for $\mathrm{C}_{16} \mathrm{H}_{11} \mathrm{~N}_{3} \mathrm{O}_{2} \mathrm{~S}$ (309.35): C, 62.12; H, 3.58; N, 13.58. Found: C, 62.17; H, 3.24; N,13.97.

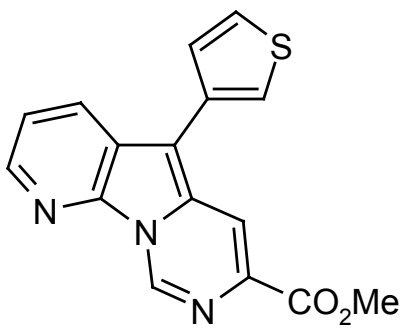

Synthesis of derivatives 15. General procedure. To a suspension of $5(1.0 \mathrm{mmol})$ in toluene/MeOH (20:1) (5 mL) was added the corresponding heteroaryl stannane (1.0 mmol) and $\mathrm{Pd}\left(\mathrm{PPh}_{3}\right)_{4}(12 \mathrm{mg}, 0.01 \mathrm{mmol})$ under argon. The mixture was heated to reflux temperature. The work-up of the reaction mixture is detailed below.

3-Methoxycarbonyl-5-(pyridin-2-yl)pyrimido[1,6-a]indole (15a). After $14 \mathrm{~h}$ reaction time the reaction mixture was filtered through Celite, the Celite washed with $\mathrm{CH}_{2} \mathrm{Cl}_{2}$ and the organic phase evaporated under reduced pressure. The residue was treated with $\mathrm{Et}_{2} \mathrm{O}$, filtered and the precipitate purified by silica gel flash chromatography in $\mathrm{CH}_{2} \mathrm{Cl}_{2}$ :acetone $(9: 1)$. Compound 15a (23\%) was isolated as a yellow solid. Mp 256-257 ${ }^{\circ} \mathrm{C}$; IR (KBr) $v_{\max } 3415$, 1726, 1585, 1459, 1431, 1371, $1222 \mathrm{~cm}^{-1} ;{ }^{1} \mathrm{H}$ NMR (500 MHz, $\left.\mathrm{CDCl}_{3}\right) \delta 3.89$ (s, 3H); 7.33 (d, $1 \mathrm{H}, J=4.6 \mathrm{~Hz})$; 7.63-7.58 (m, 2H); 7.97-7.95 (m, 2H); 8.37-8.33 (m, 1H); 8.58-8.53 (m, $1 \mathrm{H}) ; 8.78(\mathrm{~d}, 1 \mathrm{H}, J=4.9 \mathrm{~Hz}) ; 8.86(\mathrm{~d}, 1 \mathrm{H}, J=1.4 \mathrm{~Hz}) ; 9.86(\mathrm{~d}, 1 \mathrm{H}, J=1.4 \mathrm{~Hz}) ;{ }^{13} \mathrm{C} \mathrm{NMR}$ $\left(75 \mathrm{MHz} \mathrm{CDCl}_{3}\right) \quad \delta 52.8 ; 110.9 ; 111.4 ; 118.4 ; 121.0 ; 121.1 ; 122.9 ; 123.8 ; 125.9 ; 128.5 ;$ $129.2 ; 131.7 ; 135.6 ; 136.7 ; 138.4 ; 150.2 ; 153.3 ; 165.3$; MS (EI) $m / z$ (relative intensity) 303 
$\left(\mathrm{M}^{+}, 100\right) ; 244$ (29). Anal. Calcd for $\mathrm{C}_{18} \mathrm{H}_{13} \mathrm{~N}_{3} \mathrm{O}_{2}$ (303.32): C, 71.29; H; 4.33; N, 13.85 . Found: C, 71.28; H, 4.12; N, 13.85 .

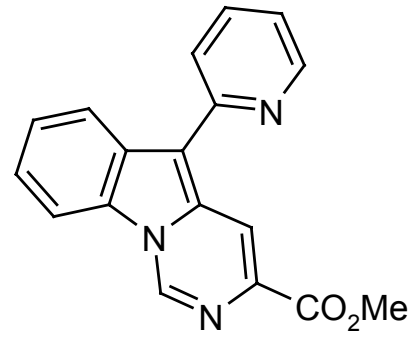

3-Methoxycarbonyl-5-(phenylethinyl)pyrimido[1,6-a]indole (15d) After $14 \mathrm{~h}$ reaction time the reaction mixture was cooled and the resulting precipitate was filtered off and washed with EtOAc. The solid was dissolved in $\mathrm{CH}_{2} \mathrm{Cl}_{2}$ and the organic solution washed with water, dried over $\mathrm{Na}_{2} \mathrm{SO}_{4}$ and filtered. The filtrate was filtered through Celite, the Celite washed with $\mathrm{CH}_{2} \mathrm{Cl}_{2}$ and the organic phase evaporated under reduced pressure to give $15 \mathbf{d}(27 \%)$ as an orange solid. Mp 255-256 ${ }^{\circ} \mathrm{C}$; IR (KBr) $v_{\max } 2190,1724,1636,1457,1436,1366,1232 \mathrm{~cm}^{-1}$; ${ }^{1} \mathrm{H}$ NMR (500 MHz, $\left.\mathrm{CDCl}_{3}\right) \delta 4.04$ (s, 3H); 7.46-7.34 (m, 3H); 7.68-7.50 (m, 4H); 8.10-8.02 $(\mathrm{m}, 2 \mathrm{H}) ; 8.48(\mathrm{~d}, 1 \mathrm{H}, J=1.5 \mathrm{~Hz}) ; 9.20(\mathrm{~d}, 1 \mathrm{H}, J=1.5 \mathrm{~Hz})$. Anal. Calcd for $\mathrm{C}_{21} \mathrm{H}_{14} \mathrm{~N}_{2} \mathrm{O}_{2}$ (326.36): C, 77.29; H, 4.32; N, 8.58. Found: C, 77.32; H, 4.39; N, 8.80.

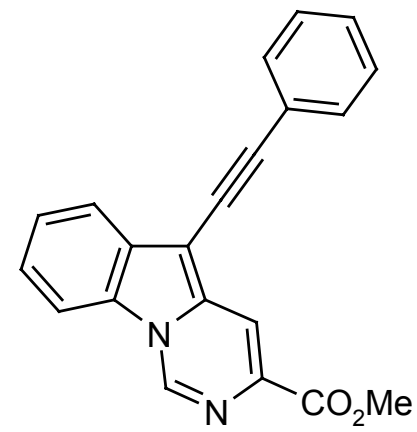

Synthesis of derivatives 16. General procedure. To a suspension of $6(1.0 \mathrm{mmol})$ in toluene/MeOH (20:1) (5 mL) was added the corresponding heteroaryl stannane (1.0 mmol) and $\mathrm{Pd}\left(\mathrm{PPh}_{3}\right)_{4}(12 \mathrm{mg}, 0.01 \mathrm{mmol})$ under argon. The mixture was heated under reflux for $14 \mathrm{~h}$ 
and then allowed to cool down to room temperature. The reaction mixture was filtered through Celite, the Celite washed with $\mathrm{CH}_{2} \mathrm{Cl}_{2}$ and the organic phase evaporated under reduced pressure. The residue was purified by silica gel flash chromatography to afford pure compounds 16.

\section{7-Methoxycarbonyl-5-(pydirin-2-yl)pyrido[3',2':4,5]pyrrolo[1,2-c]pyrimidine (16a).}

Chromatography in $\mathrm{CH}_{2} \mathrm{Cl}_{2}$ :acetone (95:5) afforded 16a (42\%) as a yellow solid. Mp 257-258 ${ }^{\circ} \mathrm{C}$; IR (KBr) $v_{\max } 3450,1715,1575,1459,1431,1370,1225 \mathrm{~cm}^{-1} ;{ }^{1} \mathrm{H}$ NMR $(500 \mathrm{MHz}$, $\left.\mathrm{CDCl}_{3}\right) \delta 4.03(\mathrm{~s}, 3 \mathrm{H}) ; 7.26(\mathrm{ddd}, 1 \mathrm{H}, J=7.9,4.9,1.3 \mathrm{~Hz}) ; 7.57(\mathrm{dd}, 1 \mathrm{H}, \mathrm{J}=8.2,4.6 \mathrm{~Hz}) ; 7.77$ $(\mathrm{d}, 1 \mathrm{H}, \mathrm{J}=7.9 \mathrm{~Hz}) ; 7.85(\mathrm{td}, 1 \mathrm{H}, J=7.9,1.8 \mathrm{~Hz}) ; 8.63(\mathrm{dd}, 1 \mathrm{H}, J=4.6,1.5 \mathrm{~Hz}) ; 8.72(\mathrm{dd}, 1 \mathrm{H}$, $\mathrm{J}=8.2,1.5 \mathrm{~Hz}) ; 8.81$ (d, 1H, $J=4.9 \mathrm{~Hz}) ; 8.94(\mathrm{~d}, 1 \mathrm{H} ; J=1.6 \mathrm{~Hz}) ; 9.69$ (d, 1H, $J=1.6 \mathrm{~Hz})$. Anal. Calcd for $\mathrm{C}_{17} \mathrm{H}_{12} \mathrm{~N}_{4} \mathrm{O}_{2}$ (304.31): C, 67.10; H, 3.97; N, 18.41. Found: C, 67.18; H, 3.77; N, 18.36.

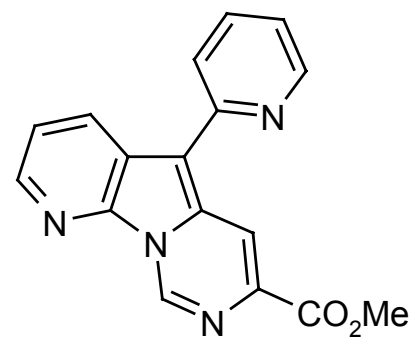

\section{5-(2-Methylthiopyrimidin-4-yl)-7-methoxycarbonylpyrido $\left[3^{\prime}, 2^{\prime}: 4,5\right]$ pyrrolo[1,2-c]-} pyrimidine (16c). Chromatography in $\mathrm{CH}_{2} \mathrm{Cl}_{2}$ :acetone (95:5) afforded $\mathbf{1 6 c}(63 \%)$ as a yellow solid. Mp 235-236 ${ }^{\circ} \mathrm{C}$; IR (KBr) $v_{\max } 3453,1712,1556,1437,1365,1336,1181 \mathrm{~cm}^{-1}$; ${ }^{1} \mathrm{H}$ NMR (500 MHz, $\left.\mathrm{CDCl}_{3}\right) \delta 2.73(\mathrm{~s}, 3 \mathrm{H}) ; 4.04(\mathrm{~s}, 3 \mathrm{H}) ; 7.41(\mathrm{~d}, 1 \mathrm{H}, J=5.4 \mathrm{~Hz}) ; 7.60(\mathrm{dd}, 1 \mathrm{H}$, $J=8.2,4.4 \mathrm{~Hz}) ; 8.57$ (d, $1 \mathrm{H}, J=5.4 \mathrm{~Hz}) ; 8.72-8.62$ (m, 2H); 9.23 (d, 1H, $J=1.5 \mathrm{~Hz}) ; 9.73$ (d, $1 \mathrm{H}, J=1.5 \mathrm{~Hz}) ;{ }^{13} \mathrm{C} \mathrm{NMR}\left(75 \mathrm{MHz} \mathrm{CDCl}_{3}\right) \delta 14.4 ; 53.2 ; 105.7 ; 113.0 ; 118.3 ; 120.8 ; 122.4$; $129.9 ; 133.7 ; 138.5 ; 138.8 ; 141.4 ; 145.1 ; 157.3 ; 160.2 ; 164.7 ; 173.2 ;$ MS (EI) $m / z$ (relative 
intensity) $351\left(\mathrm{M}^{+}, 100\right) ; 291$ (75); 246 (41). Anal. Calcd for $\mathrm{C}_{17} \mathrm{H}_{13} \mathrm{~N}_{5} \mathrm{O}_{2} \mathrm{~S}$ (351.39): C, 58.11; H, 3.73; N, 19.93. Found: C, 58.14; H, 3.71; N, 19.94.<smiles>CC(=O)c1cc2c(-c3ccnc(C)n3)c3cccnc3n2cn1</smiles>

7-Methoxycarbonyl-5-(phenylethynyl)pyrido[3',2':4,5]pyrrolo[1,2-c]pyrimidine (16d). Chromatography in $\mathrm{CH}_{2} \mathrm{Cl}_{2}$ :acetone (98:2) afforded 16d (38\%) as a orange solid. Mp 218-220 ${ }^{\circ} \mathrm{C}$; IR (KBr) $v_{\max } 3422,2923,2197,1717,1458,1434,1365,1290,1233 \mathrm{~cm}^{-1}$; ${ }^{1} \mathrm{H}$ NMR (500 $\left.\mathrm{MHz}, \mathrm{CDCl}_{3}\right) \delta 4.03(\mathrm{~s}, 3 \mathrm{H}) ; 7.50-7.38(\mathrm{~m}, 3 \mathrm{H}) ; 7.69-7.53(\mathrm{~m}, 3 \mathrm{H}) ; 8.35(\mathrm{dd}, 1 \mathrm{H}, J=7.9,1.6$ $\mathrm{Hz}) ; 8.47(\mathrm{~s}, 1 \mathrm{H}) ; 8.61(\mathrm{dd}, 1 \mathrm{H}, J=4.7,1.6 \mathrm{~Hz}) ; 9.59$ (s, $1 \mathrm{H})$. Anal. Calcd for $\mathrm{C}_{20} \mathrm{H}_{13} \mathrm{~N}_{3} \mathrm{O}_{2}$ (327.35): C, 73.39; H, 4.00; N, 12.84. Found: C, 73.35; H, 4.12; N, 12.81.

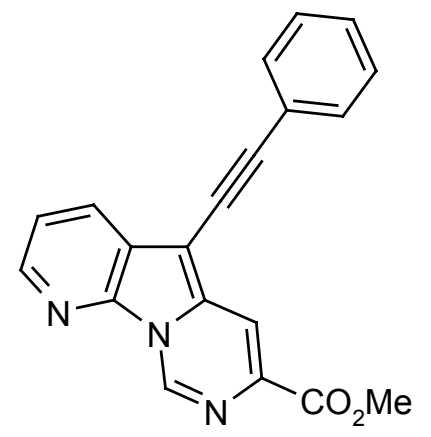

